\title{
A novel method to measure ion density in ICF experiments using $X$-ray spectroscopy of cylindrical tracers
}

\author{
G. Pérez-Callejo, ${ }^{1}$ a) M. A. Barrios, ${ }^{2}$ D. A. Liedahl, ${ }^{2}$ M. B. Schneider, ${ }^{2}$ O. Jones, ${ }^{2}$ O. Landen, ${ }^{2}$ R. L. Kauffman, ${ }^{2}$ \\ L. J. Suter, ${ }^{2}$ J. D. Moody, ${ }^{2}$ S. J. Rose, ${ }^{1,3}$ and J. S. Wark ${ }^{1}$ \\ 1) Department of Physics, Clarendon Laboratory, University of Oxford, Parks Road, Oxford OX1 3PU, UK \\ ${ }^{2)}$ Lawrence Livermore National Laboratory, Livermore, California 94550, USA \\ 3) Plasma Physics Group, The Blackett Laboratory, Imperial College London, Prince Consort Road, London, SW7 \\ 2AZ, UK
}

(Dated: 26 October 2020)

The indirect drive approach to inertial confinement fusion (ICF) has undergone important advances in the past years. The improvements in temperature and density diagnostic methods are leading to more accurate measurements of the plasma conditions inside the hohlraum and therefore to more efficient experimental designs. The implementation of dot spectroscopy has proven to be a versatile approach to extracting spaceand time-dependent electron temperatures. In this method a microdot of a mid- $\mathrm{Z}$ material is placed inside the hohlraum and its K-shell emission spectrum is used to determine the plasma temperature. However, radiation transport of optically thick lines acting within the cylindrical dot geometry influences the outgoing spectral distribution in a manner that depends on the viewing angle. This angular dependence has recently been studied in the high energy density (HED) regime at the OMEGA laser facility, which allowed us to design and benchmark appropriate radiative transfer models that can replicate these geometric effects. By combining these models with the measurements from the dot spectroscopy experiments at the National Ignition Facility (NIF), we demonstrate here a novel technique that exploits the transport effects to obtain time-resolved measurements of the ion density of the tracer dots, without the need for additional diagnostics. We find excellent agreement between experiment and simulation, opening the possibility of using these geometric effects as a density diagnostic in future experiments.

PACS numbers: Valid PACS appear here

Keywords: X-Ray Spectroscopy, Anisotropy, Geometry, Optical Depth, Fusion

\section{INTRODUCTION}

The evolution of the plasma inside hohlraums in inertial confinement fusion (ICF) experiment ${ }^{1 / 3}$ is extremely complicated to model, owing to the interactions between the laser drive, the gold plasma from the hohlraum walls, the fill gas, the Planckian X-ray field, and the fuel capsule. Modelling the evolution of these processes, and how they affect the conditions inside the hohlraum during an ICF implosion, requires a deep understanding of laserplasma interactions and computationally expensive multidimensional radiative hydrodynamic simulations. On top of that, the results from those simulations are model dependent ${ }^{4}$, and experimental data that can help benchmark them are scarce.

Given the great amount of information that can be extracted from the X-ray spectra of plasmas, X-ray spectroscopy is a fruitful approach to characterizing the plasma conditions in ICF and it can be used to estimate the temperature distribution in a plasma ${ }^{5-12}$. In particular, the dot spectroscopy campaign $\frac{13 \mid 14}{}$ comprises a series of experiments that were carried out at the the National Ignition Facility ${ }^{\sqrt{15}}$ (NIF) with the aim of using K-shell spectroscopy to diagnose the evolution of the electron

\footnotetext{
a)gabriel.perezcallejo@physics.ox.ac.uk
}

temperature inside a hohlraum during an ICF implosion.

The idea of the dot spectroscopy method has been around for several decades ${ }^{78}$. It had already been implemented for diagnosing the conditions inside hohlraums in indirect drive ICF in the late 1990s at the NOVA laser facility (precursor of NIF) ${ }^{11112}$, but only recently have dot-based experiments been conducted at the NIF $13 \mid 14$. The key feature of this campaign is the addition of a mid-Z tracer dot in the hohlraum. At the temperatures and densities characteristic of high energy density (HED) plasmas, the tracer gets highly ionized and starts emitting K-shell X-rays. The dot materials $(\mathrm{Z} \sim 23-38)$ were chosen so that their $\mathrm{K}$-shell emission was in range of $5-9 \mathrm{keV}$, a region in which emission from the hohlraum $\mathrm{Au})$ is bound-free continuum. Thus, the tracers' spectra can be easily resolved.

Hydrodynamic simulations using the code HYDRA ${ }^{16}$ show that the dynamics of the dots (initially: radius $\sim 400 \mu \mathrm{m}$ and thickness $\sim 0.3 \mu \mathrm{m}$ ) do not alter the conditions inside the hohlraum significantly, and that the temperature and electron density of the dots' plasma are indicative of those of the hohlraum plasma surrounding it. Therefore, by time-resolving the K-shell line emission of the tracer dot, we can observe the temperature evolution inside the hohlraum during the implosion $\frac{13}{2}$. Additionally, if the position of the dot is also measured, one can link the temperature evolution to a particular region of the hohlraum. This has allowed different parts 


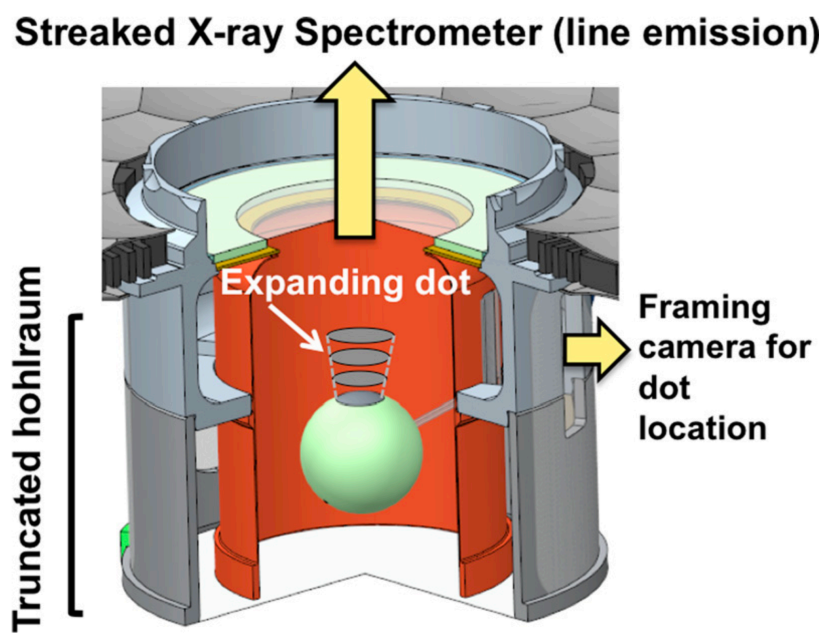

(a)

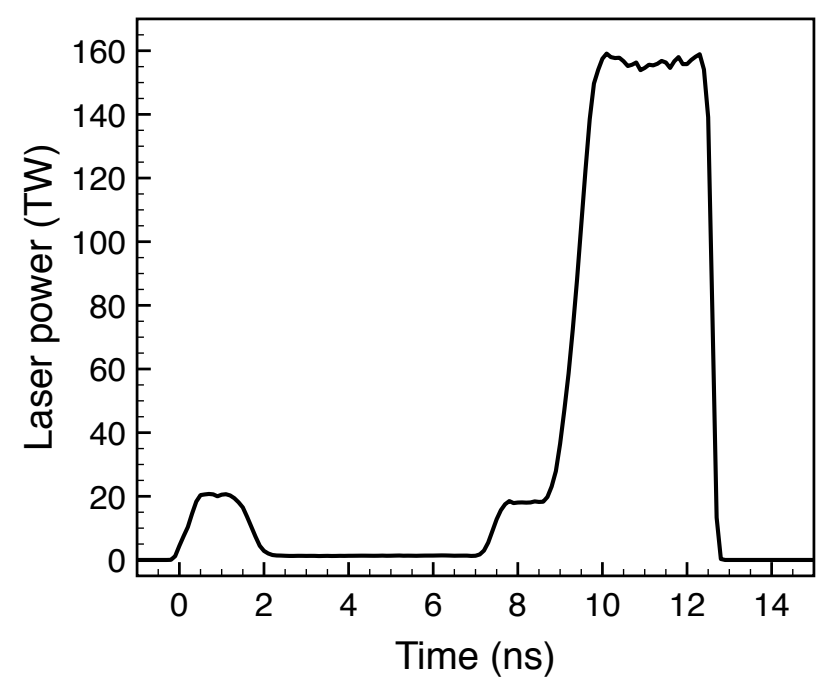

(b)

FIG. 1. 1a Schematic of the experimental set-up showing the view of the different instruments and the position of the dot and the capsule inside the truncated hohlraum. Reproduced from Barrios, M. A. et al. "Electron temperature measurements inside the ablating plasma of gas-filled hohlraums at the National Ignition Facility." Physics of Plasmas vol. 23, no. 5 (2016), with the permission of AIP Publishing. (1b) The 12.7 ns high-foot pulse that was used in the experiment.

of the hohlraum to be mapped, providing a experimental platform to study heat transport in indirect drive ICF ${ }^{14}$. Nevertheless, no measurements of the electron density of the dots have yet been reported.

However, analyzing the K-shell emission of the dots is non-trivial. It was shown by Kerr et al! $\frac{17}{19}$ that in nonspherical plasmas the intensites of optically thick components of the X-ray spectra are enhanced relative to the optically thin limit if the line of sight through the plasma is shorter than the mean chord of the emitting volume. Conversely, if the line of sight length exceeds the mean chord length, their intensites are reduced. While the line of sight depends on the angle at which the detector views the source, the mean chord $\hat{l}$ is a fundamental property of a given geometry and thus, the ratio from the line of sight to the mean chord varies as a function of the angle of observation. The mean chord of a convex body can be easily calculated by means of the Dirac-Fuchs theorem 20121 as

$$
\hat{l}=\frac{4 V}{S},
$$

where $V$ is the volume of the body and $S$ its surface area. For an infinite planar source of thickness $\Delta z$ this equation returns the well-known value of $\hat{l}=2 \Delta z$ (which corresponds to the chord at $60^{\circ}$ from the normal of the plane). Therefore, the need arises of accounting for the angular dependence of the spectral distribution which plays directly into experimental design considerations and spectral analysis. This effect has previously been observed in astrophysical and laboratory plasmas $22 \mid 23$, and several methods have been proposed to diagnose the geometry of astrophysical bodies by studying it ${ }^{1824}$.
Over the past few years several experiments at the OMEGA laser facility 25 were dedicated to measuring these geometric effects in HED cylindrical plasmas ${ }^{26}$ with the aim of developing accurate models that can be applied to the K-shell spectra of the aforementioned tracer dots. The geometric effects on the spectra were assessed by focusing on the ratio of the resonance $\left(1 s 2{ }^{1}{ }^{1} P_{1} \rightarrow\right.$ $\left.1 s^{2}{ }^{1} S_{0}\right)$ to the intercombination $\left(1 s 2 p{ }^{3} P_{1} \rightarrow 1 s^{2}{ }^{1} S_{0}\right)$ lines of the He $\alpha$ emission (hereafter noted the $w$ and $y$ lines respectively, following Gabriel's notation ${ }^{27}$ ). It was shown that two-dimensional radiative transfer simulations using the code Cretin ${ }^{28}$ can reproduce the experimental line ratios to a much higher degree than models which do not include the plasma geometry. Additionally, it was found that the $w / y$ ratio obtained from two different views of the cylinder can be used as a diagnostic of the ion density of the plasma ${ }^{2629}$, given that these ratios are dictated by the optical depth of the plasma through the mean chord and the line of sight ${ }^{18 \mid 19 / 30}$, which is directly proportional to the ion density.

In this paper, we combine the predictions from the Cretin model for cylindrical plasmas that was benchmarked at the OMEGA laser in the aforementioned experiments 26 , with the time-resolved $w / y$ ratios measured in the dot spectroscopy experiments at the NIF. We show how these ratios, combined with measurements of the thickness of the dot and the assumption that the dot stays cylindrical, can be used to retrieve timeresolved values of the ion density of the plasma inside the hohlraum at the position of the tracer dots, by studying two particular shots with Mn cylindrical tracers. The predictions from hydrodynamic simulations us- 


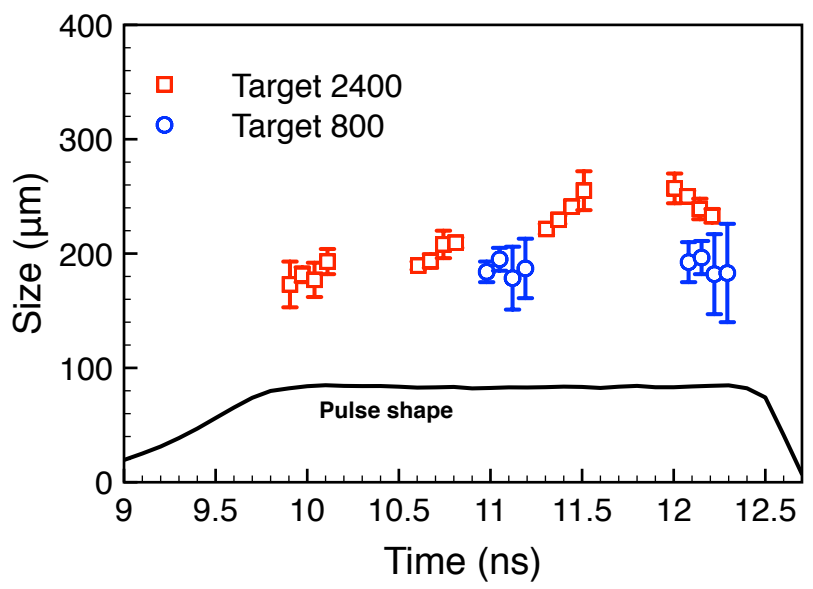

(a)

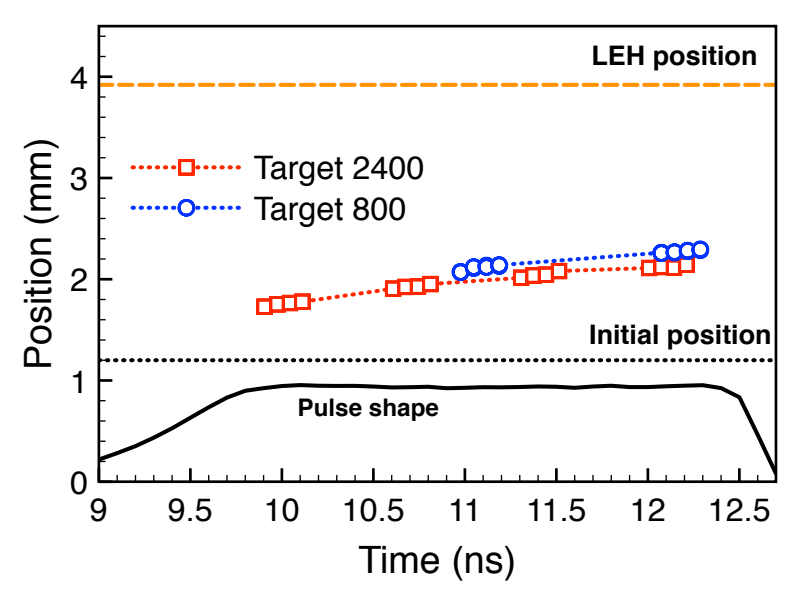

(b)

FIG. 2. 2a Thickness of both Mn dots measured with the XRPINH as a function of time. 2b Position of the dots across the hohlraum with respect to the LEH and their original position. The error bars are contained within the symbol size.

ing the code HYDRA are in good agreement with the results for the ion density obtained using this method. Furthermore, our obtained results are consistent with all the data from the experiment. Finally, we show how if the cylindrical geometry of the tracers is neglected, and a simple spherically symmetric plasma is assumed, the same method yields results for the ion density that differ in up to an order of magnitude from those that include the cylindrical geometry, and the consistency with the rest of the data is lost.

As mentioned above, the electron density of the dots' plasma is indicative of that of the plasma inside the hohlraum, and given that the electron density of the dots' plasma is directly related to the ion density $\left(n_{e}=\bar{Z} n_{i}\right)$, measuring the ion density of the dots is of great importance to diagnosing the evolution of the electron density inside the hohlraum. This work opens up the possibility of using the dot spectroscopy technique to jointly map the temperature and density of the plasma in different regions of the hohlraum as a function of time by using cylindrical tracers that act as a surrogate of the plasma inside the hohlraum.

\section{EXPERIMENTAL SET-UP}

The experiments in the dot spectroscopy campaign were performed at the NIF laser. The Viewfactor hohlraum ${ }^{31}$, which is a hohlraum truncated at $\sim 75 \%$ of its length, was used in order to eliminate background $\mathrm{X}$-rays that would be produced by a real ICF capsule and the flange of the lower laser entrance hole (LEH) of a real hohlraum. To maintain surrogacy of plasma conditions in the equivalent hohlraum in the upper LEH region, a thin parylene $(\mathrm{CH})$ spherical shell $(25 \mu \mathrm{m}$ thick, $2.4 \mathrm{~mm}$ in diameter) replaces the ICF fuel capsule. The hohlraums were $4.69 \mathrm{~mm}$ in diameter and $5.92 \mathrm{~mm}$ long, with a LEH
$3.1 \mathrm{~mm}$ in diameter in the non-truncated base. The capsule was centered $3.92 \mathrm{~mm}$ directly below the LEH and aligned with the axis of the hohlraum. The hohlraums were filled with neopentane $\left(\mathrm{C}_{5} \mathrm{H}_{12}\right)$ at $1.37 \mathrm{mg} / \mathrm{cm}^{3}$ and positioned in the NIF target chamber such that the center of the $\mathrm{CH}$ capsule was at the center of the chamber. This set-up is shown in figure 1 a.

A Mn tracer dot was sputter-coated on top of the capsule keeping the axis of the dot aligned with the axis of the hohlraum. We focus on two particular shots in which the experimental conditions were nominally identical (and identical to those in Barrios et al. ${ }^{[13}$ ), with the exception of the thickness of the dots. In shot N150913002 the thickness of the dot was $2400 \AA$, whereas in shot N151116-003 the thickness was $800 \AA$, both dots made of pure manganese. These targets will hereafter be referred to as Target 2400 and Target 800 respectively. Both dots had a radius of $400 \mu \mathrm{m}$

While the K-shell emission of the dots was observed with the absolutely calibrated NIF X-ray Spectrometer $(\mathrm{NXS})^{32}$ looking down the hohlraum axis through the LEH, the position and axial expansion of the dots were measured with an X-Ray Pinhole Camera (XRPINH). The XRPINH viewed the dot through a rectangular aperture $(250 \mu \mathrm{m} \times 2300 \mu \mathrm{m})$ cut into the hohlraum wall, as shown in figure 1a. Another aperture $(400 \mu \mathrm{m} \times 2000 \mu \mathrm{m})$ was cut in the opposite wall. This aperture was wider to avoid background from glowing gold walls, and shorter to provide a fiducial in axial direction. Both apertures had $80 \mu \mathrm{m}$ thick polyimide windows. Additionally, a Static $\mathrm{X}$-ray Imager (SXI) positioned below the hohlraum was used to obtain a time-integrated image of the plasma inside the hohlraum.

The laser profile used in the experiment, shown in figure $1 \mathrm{~b}$, was a $12.8 \mathrm{~ns}$ high-foot pulse, delivering $\sim 600 \mathrm{~kJ}$ of $3 \omega$ light energy in the hohlraum with a peak power of $160 \mathrm{TW}$ for $\sim 3 \mathrm{~ns}$. Due to the truncated geometry 
of the hohlraum, the outer beams (impinging at $44.5^{\circ}$ and $50^{\circ}$ ) from the lower hemisphere could not be used, so only 128 out of the 192 beams available were implemented. More details on the experimental set-up for this campaign can be found in Barrios et al. ${ }^{[3]}$, where these experiments were first reported.

\section{EXPERIMENTAL RESULTS}

The XRPINH data was used to measure the thickness of the targets along their expansion axes. This is shown in figure 2a where the blue circles represent the Target 800 data, whereas the red squares correspond to Target 2400. This color convention will be used for the remainder of this paper. The error bars correspond to the variation in the thickness measured across the aperture. For Target 800 half as many values as for Target 2400 were measured, as in this case the frames in the XRPINH were timed in pairs, so two measurements were taken at each timestep. The thickness of the dots does not change noticeably during the peak power of the laser pulse (between 9.5 and $12.5 \mathrm{~ns}$ ), although they have expanded axially by a factor of $\sim 10^{3}$ in the first $9 \mathrm{~ns}$, considering their original thicknesses.

For the remainder of this paper, we will assume the thickness of each dot to be constant during the peak power of the laser pulse, with values:

$$
\begin{aligned}
& H_{2400}=220 \pm 30 \mu \mathrm{m}, \\
& H_{800}=187 \pm 14 \mu \mathrm{m} .
\end{aligned}
$$

Note that both thicknesses are within $20 \%$ of each other, whereas the initial thicknesses of the dots differed by a factor of 3. This is in agreement with the predictions from the HYDRA simulations.

The position of the dot along the hohlraum axis, measured with the XRPINH and shown in figure 2b also gives important information, as it allows one to know which part of the hohlraum is being diagnosed by the dots, as they trace the ablation front of the $\mathrm{CH}$ capsule. Although in the two shots that are being analysed the dots were coated on top of the capsule, there have been extensions to this campaign in which the dots were placed closer to the LEH or on the equator of the capsule to diagnose different regions of the hohlraum 14 . In this case, as the dots are coated on top of the $\mathrm{CH}$ capsule, the ablation pressure pushes the dot outward until it is eventualy balanced by the pressure from the hot plasma of the laser deposition region, the point at which the velocity of the dot is reduced. Figure $2 \mathrm{~b}$ shows that, as expected, both dots move to similar positions inside the hohlraum, never reaching the regions close to the LEH. The error bars in this plot are contained within the size of the symbols.

Good quality time-resolved spectral data were obtained for both targets. Figure 3 shows the streaked K-shell spectrum of Target 800 together with the laser pulse. The $\mathrm{x}$-axis shows the time with respect to the start of the pulse, whereas the y-axis corresponds to the energy of the emitted photons. The main visible features

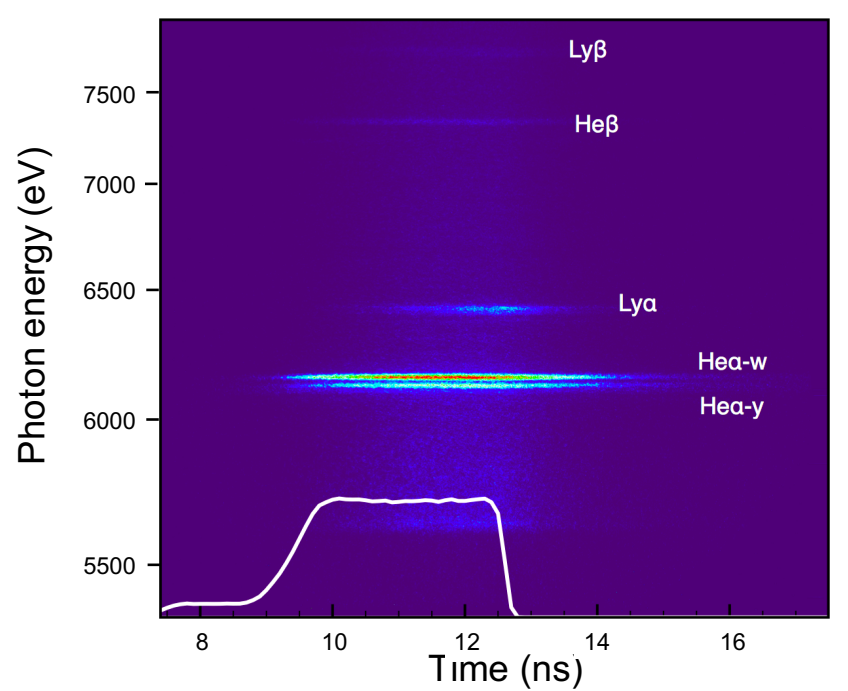

FIG. 3. Streak-camera spectrum for Target 800. The laser pulse profile is shown in white. As the plasma evolves, it can be seen that the relative fluxes from the $\mathrm{He} \alpha(\sim 6200 \mathrm{eV})$ and Ly $\alpha(\sim 6500 \mathrm{eV})$ components change.

correspond to the He $\alpha$ complex $(\sim 6200 \mathrm{eV})$, and the Ly $\alpha(\sim 6500 \mathrm{eV}), \mathrm{He} \beta(\sim 7300 \mathrm{eV})$ and $\mathrm{Ly} \beta(\sim 7600 \mathrm{eV})$ lines, indicated in the figure. Note how during the rise of the pulse there is not any K-shell emission, as it starts when the pulse reaches peak power. Focusing in particular on the brighter $\operatorname{He} \alpha$ and $\operatorname{Ly} \alpha$ emission, note that, as the $\operatorname{Ly} \alpha$ line is indicative of a high plasma temperature (it requires a higher ionisation state to be produced) its emission starts later in time than that of the He $\alpha$ complex, when the plasma reaches a higher temperature. The inverse effect is observed after the laser pulse ends and the plasma starts to cool, with the Ly $\alpha$ emission fading out first.

\section{SPECTRAL ANALYSIS}

The spectra obtained with the NXS are continuous in time, and with a resolution of $15-20 \mathrm{eV}$ in the spectral range of consideration. The temporal resolution of the streak camera is $\sim 80 \mathrm{ps}$, with $2.4 \mathrm{ps} /$ pixel, however, to minimize the signal-to-noise ratio, the spectra were integrated in windows of 130 pixels, equivalent to $312 \mathrm{ps}$.

In this experiment, the dots' plasma is placed between the $\mathrm{CH}$ plasma from the capsule and the neopentane plasma from the gas fill of the hohlraum. HYDRA simulations predicted that the gold plasma from the walls does not expand enough to get in contact with the dots' plasma. This was further confirmed by the SXI images. Therefore, the Mn K-shell emission from the dots does not experience any radiation transport effects once it escapes the dot (as it only has to travel through the neopentane gas, which is transparent for the photon energies 


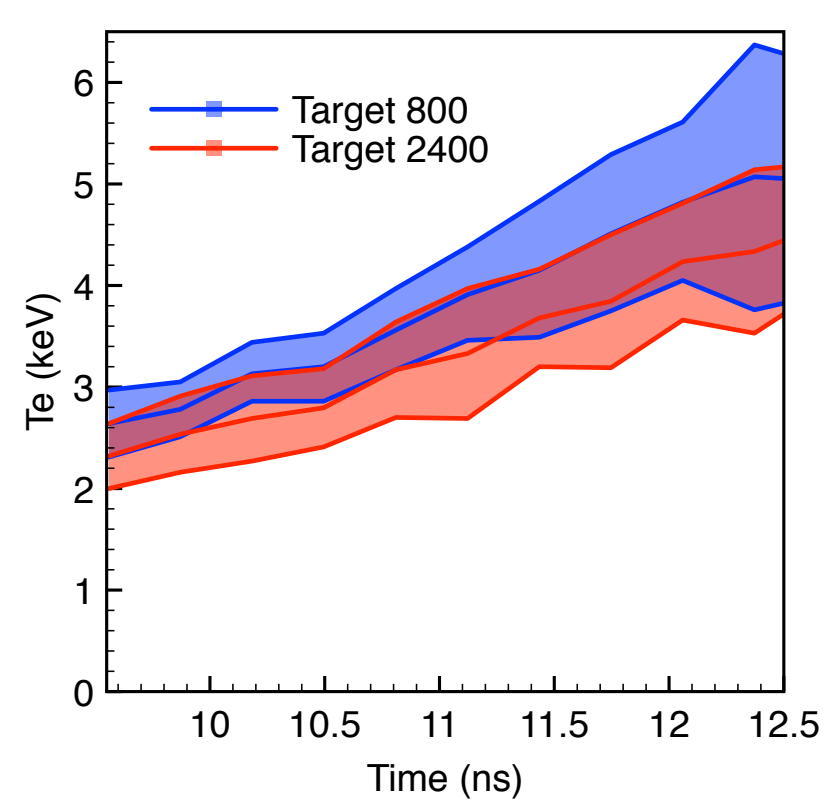

FIG. 4. Width of the temperature distributions as a function of time for both Target 800 and Target 2400. Both targets evolve with similar temperatures (within 20\%), despite their different initial thicknesses. The vertical extent of the shaded area corresponds to a standard deviation of the temperature distribution and should not be taken as an error bar. Ten spectra were used to produce this figure, each of them averaged over $312 \mathrm{ps}$.

involved).

At the experimental conditions $\left(T_{e} \sim 1-6 \mathrm{keV}\right.$ and $n_{e} \sim 10^{21} \mathrm{~cm}^{-3}$ ), the line ratios in the K-shell emission spectrum are good indicators of the plasma temperature as, except for the $w$ component of the He $\alpha$ complex, the other observed lines ( $y$ line, $\operatorname{Ly} \alpha, \operatorname{He} \beta$ and $\operatorname{Ly} \beta$ ) are optically thin. Around $2 \mathrm{keV}$, the optical depth of the $\mathrm{He} \beta$ line is $\sim 1$, but as it decreases with temperature, we do not consider this to be significant. On the other hand, these ratios are relatively insensitive to density variations 33 . In fact, the main effects of density variations in this regime are modifications of the line widths, which are unnoticeable in this case owing to the spectral resolution of the NXS. This sensitivity to temperature and insensitivity to density, makes the Mn K-shell spectra a good temperature diagnostic.

In previous work ${ }^{13}$, it was found that the single temperatures obtained from individual line ratios could not reproduce all the spectral features, with variations of $\sim 200 \mathrm{eV}$ being necessary to reproduce all the components of the spectra. To fix this, we obtained the differential emission measure (dEM) of the spectra. This approach consists on assuming that the plasma has different regions at slightly different temperatures such that the observed spectrum $S$ consists of a weighted composition of the different temperatures present in the plasma as

$$
S=\int_{T_{\min }}^{T_{\max }} d T w(T) S(T) \sim \sum_{T_{\min }}^{T_{\max }} w(T) S(T),
$$

where $S(T)$ are the spectra from a uniform plasma at a temperature $T$, and $w(T)$ are the weights associated to each single-temperature spectrum. These weights are related to the relative size of the region at that particular temperature.

To determine the weight values and get an estimate of the distribution of temperatures within the plasma, we used a genetic algorithm ${ }^{34}$ to fit the full experimental spectra, following an identical procedure to that described in Marley et al ${ }^{33}$. The algorithm works with a previously-generated grid of optically thin singletemperature spectra $S(T)$ at a constant electron density of $n_{e}=10^{21} \mathrm{~cm}^{-3}$ produced with the 0 -dimensional code SCRAM $^{35}$ (no density variations were included in the $\mathrm{dEM}$, given that the spectra is mostly insensitive to density in this regime). The reason why no optical depth is included in the spectral grid is that, as mentioned above, radiative transfer effects on the spectra depend on the exact geometry of the plasma, and the radii of the targets are unknown (as they were not measured in the experiment). Therefore, generating a spectra grid including radiative transfer effects at this stage would bias the obtained temperature distribution. Given that the $w$ line is the only optically thick component of the spectra, its emission was removed to avoid the influence of its optical depth on the result from the fit. Removing this line does not affect the temperature calculation much, as information can be gathered from the relative emission from the rest of the lines shown in figure 3 .

Initially, the genetic algorithm generates several random temperature weigths and selects those who produce a spectrum that fits the data best. The next iteration of the algorithm takes the best combinations from the previous iteration, and combines them (hence the name 'genetic'). Every iteration, the newly generated combinations, or offspring, can have some of the weights randomly changed, or 'mutated'. By allowing the algorithm to iterate several thousands of times, the temperature distribution eventually converges to the best possible fit the algorithm can find. Note that this approach can only provide information about the relative abundance of different temperature regions within the plasma, but it fails to provide any information as to how these temperatures are distributed (wheter axially, radially or both). Additional uncertainty arises from the finite resolution of the grid of single-temperature spectra, which in this case was $100 \mathrm{eV}$

However, it is not technically possible to ensure that the temperature distribution found by the genetic algorithm is unique. In fact, the error bars of the data can yield several local minima for the loss function of the fit within the same temperature bin. It is therefore necessary to run this algorithm several times for each case in 


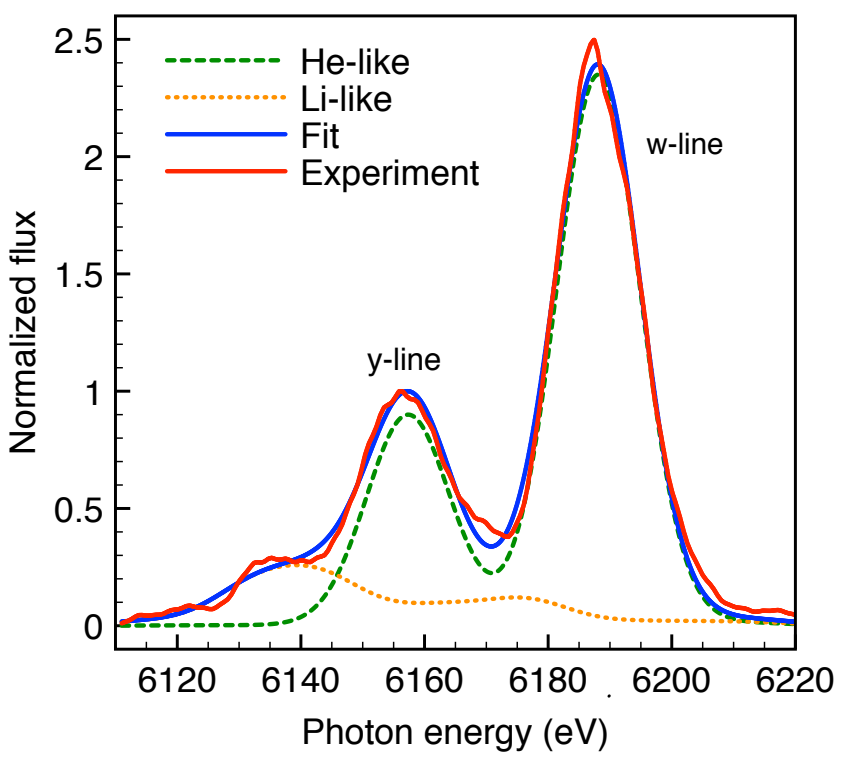

(a)

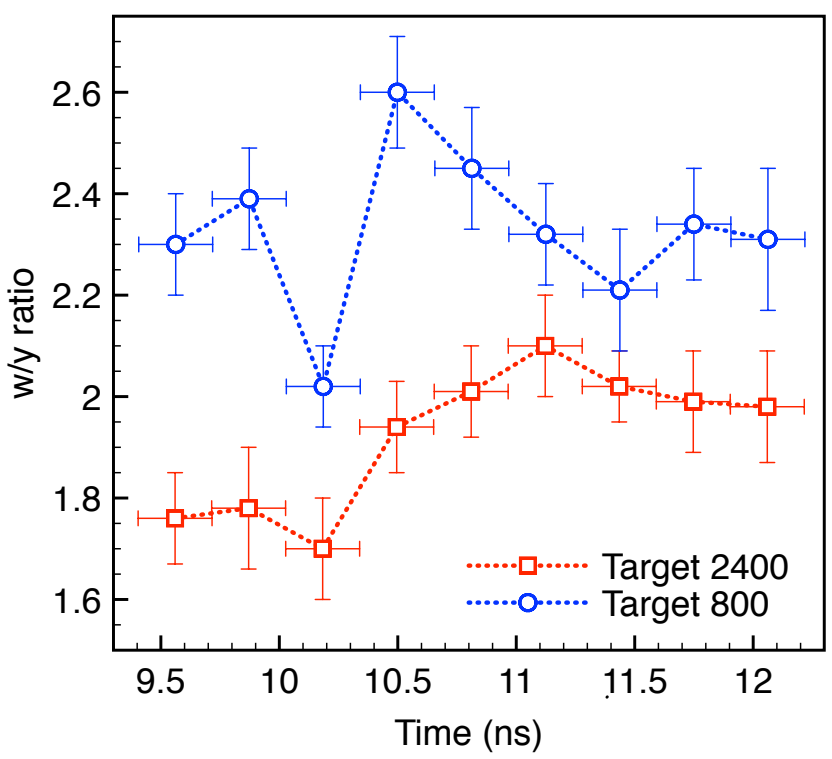

(b)

FIG. 5. 5a Example of the fitting process. The Li-like satellites are modelled using the code Cretin 28 , and the $w$ and $y$ lines are fitted to gaussian profiles to obtain their ratio. (5b) Results for the $w / y$ ratio for both targets as a function of time, obtained from the fitting procedure. The ratio for Target 800 is consistently larger than for Target 2400, which is indicative of the dots having different mean chords (see section II). The horizontal error bars correspond to the width of the time-integrating window used to analyse the spectral data, whereas the vertical error bars correspond to the standard deviation in the line ratios obtained from the fitting routine.

order to explore the parameter space and gain confidence on the result.

Each time the algorithm is initiated, the initial temperature distributions generated are random and, additionally, the way in which the best-fitting distributions are combined, and the mutations that are introduced are also random. This combination of random choices makes the code evolve following a completely different path every time it is run. Therefore, arriving at the same result (within uncertainty), decreases the probability of this solution being non-unique exponentially with the number of times the algorithm is run. By running the algorithm 5-6 times, this probability can be lowered to about $\sim 1 \%$.

We found that the central temperatures of our obtained solutions lie within the expected temperature range, and in fact, agree with the results previously published by Barrios et al.13114. In fact, the width of the temperature distribution obtained by the genetic algorithm lies within the error bars of the results by Barrios et al., providing further confidence in the width of the distribution obtained by our algorithm.

Note that the central temperatures of both targets agree within $\sim 20 \%$, and the distributions overlap for the whole duration of the laser pulse. This is consistent with the hypothesis that the dot equilibrates with its surroundings, and thus the temperature from the dot is indeed the temperature of the plasma at the corresponding region of the hohlraum, validating its role as a surrogate.
Additionally, we did a detailed fit of the time-resolved emission from the He $\alpha$ complex to extract the energyintegrated $w / y$ ratio as a function of time. To do so, we model the Li-like emission using a 0-dimensional approach, and remove their contribution from the spectra. This can be done because the Li-like satellite lines are extremely optically thin in this regime (their optical depth is $\sim 0.1$ ), and therefore are not affected by radiative transfer effects. Additionally, for temperatures above $2 \mathrm{keV}$, such as in this case, their emission is very weak and therefore the uncertainty introduced by removing their contribution is negligible. This way, the only remaining components of the He $\alpha$ complex are the $w$ and $y$ lines (with optical depths of $\sim 8$ and $\sim 0.6$ respectively), whose emission can easily be separated and integrated to obtain the line ratio.

An example of these detailed fits, together with the results for the line ratio are shown in figure 5 . It can be seen that Target 800 shows a consistently greater $w / y$ ratio than Target 2400 . This is a result of the difference in the initial thickness of the dots. As discussed in section I the line ratios depend on the line of sight and the mean chord of the plasma. In this particular case, both dots have similar thicknesses during the time that the laser pulse is at peak power, and therefore, the lines of sight are comparable in both cases. However, that is not necessarily the case with the mean chords (which depend on the thickness and radius of the cylinder), given that the radial expansion of the dots might be different, ow- 
ing to the initial differences between them. Therefore, differences in the radial expansion of the dots, translate to differences in their mean chords that affect the line ratios, producing the differences shown in figure 5b. In both cases, there is a spike in the line ratios around $10.2 \mathrm{~ns}$, caused by an artifact in the streaked spectra.

By assuming the targets to remain perfectly cylindrical, their radius and density can be related to the known thickness of each plasma as

$$
R\left(n_{i}\right)=\sqrt{\frac{N}{\pi H n_{i}}}
$$

where $R$ is the dot's radius, $H$ is the dot's thickness (values from figure 2a) and $N$ is the total number of ions in the dot plasma. For each of the targets considered in this paper $N$ is known and equal to

$$
N(i)=N_{A} \cdot \frac{\rho_{M n}}{M_{M n}} \pi H_{0}(i) R_{0}^{2},
$$

where $i$ is used to identify the targets (either Target 800 or Target 2400), $N_{A}$ is Avogrado's number, $\rho_{M n}$ and $M_{M n}$ are the solid density and atomic weight of manganese respectively, and $H_{0}(i)$ and $R_{0}$ are the initial thickness and radius of each target (note that $R_{0}$ does not depend on the target as both dots had the same initial radius $R_{0}=400 \mu \mathrm{m}$ ).

This assumption, although not ideal, reflects the fact that the gold plasma from the hohlraum walls does not get mixed with the dot. Additionally, given that the only source of $\mathrm{Mn}$ ions in the hohlraum is the dot, by linking the radius of the cylinder to the density of $\mathrm{Mn}$ ions through the measured thickness, we make sure that the possibility of radial expansion of the dot is included consistently with the rest of the variables. We make the safe assumptions that the total number of Mn particles in the dot is constant, and that the entire particle population is always in the field of view of the NXS, which are direct consequences of assuming a perfect cylinder. Under these assumptions the number $N(i)$ remains constant through the expansion in both cases.

Using the values of $H$ measured from the XRPINH data for each target and the relation between the radius of a cylinder and the ion density (from equation 3), we performed simulations using the cylindrical Cretin model developed and benchmarked in recent OMEGA experiments for HED plasmas 26 . By generating a grid of simulations, each at a uniform temperature and ion density (therefore uniform radius, as given by Equation 3), we can produce a 'map' of the expected $w / y$ ratio for each of the targets as a function of these conditions, assuming a perfectly cylindrical geometry.

As the experimental $w / y$ ratios and temperatures are known (figures 5b and 4 respectively), it is now possible to compare the experimental values with the results from the cylindrical simulations. Figure 6 shows the 'map' of the $w / y$ ratio of a perfect cylinder as a function of temperature and ion density for Target 2400 (for Target

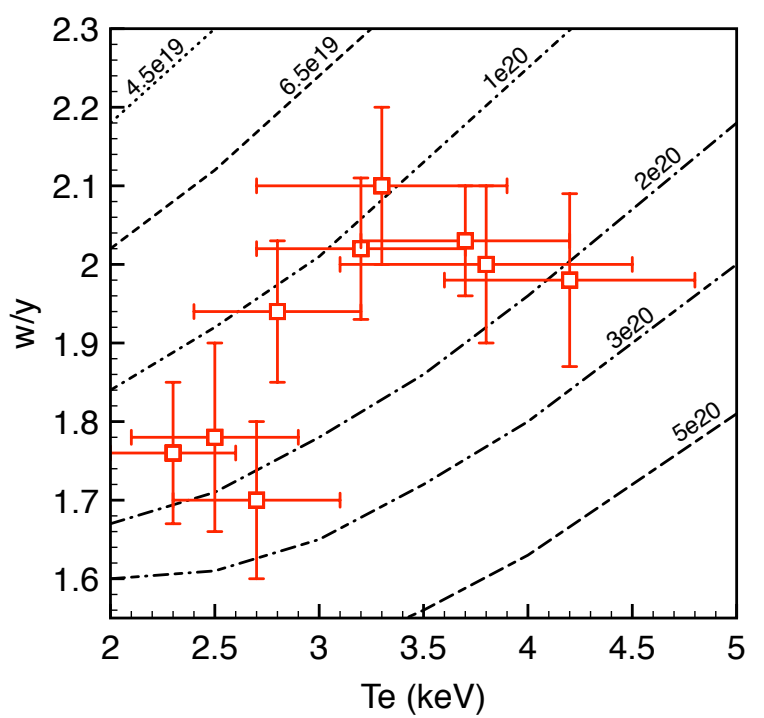

FIG. 6. Map of the calculated $w / y$ ratio for Target 2400 as a function of the plasma temperature and ion density. The red squares correspond to the $w / y$ ratios from the experiment, plotted against temperature. Each black line corresponds to the line ratios from the Cretin model for a given ion density as a function of temperature. Note that each iso-density line corresponds to a fixed radius of the cylinder, given by equation 3 to conserve the total number of ions in the dot. These lines are labelled with the corresponding ion density for clarity.

800 , a different map is obtained as the values of $N$ and $H$ are different). The x-axis corresponds to temperature, whereas the $\mathrm{y}$-axis shows the $w / y$ ratio. Each of the black lines across the plot corresponds to a single and fixed ion density, indicated by the labels. The red squares represent the experimental data with the corresponding uncertainty regions (corresponding to the width of the temperature distribution and the error in the $w / y$ fit). Note that time is not explicitly plotted in this figure, but given that the temperature increases monotonically during the peak power of the pulse (as shown in figure 4), moving up in temperature is equivalent to moving later in time.

\section{RESULTS FOR THE PLASMA DENSITY}

From the map shown in figure 6 (and the equivalent result for Target 800), a value of $n_{i}$ with an associated uncertainty can be interpolated for each of the experimental $\left(T_{e}, w / y\right)$ points. These density values are shown in figure 7 together with the predictions from 2-dimensional hydrodynamic simulations run prior to the experiment, obtained with the code HYDRA ${ }^{16}$. Although these simulations were run for a $3200 \AA$ thick dot, the ion density values are comparable, as the HYDRA prediction for the position of the dot is similar to the measured trajectory of the dots of study (shown in figure 2b), and therefore the simulated dot samples a similar region of the hohlraum as 


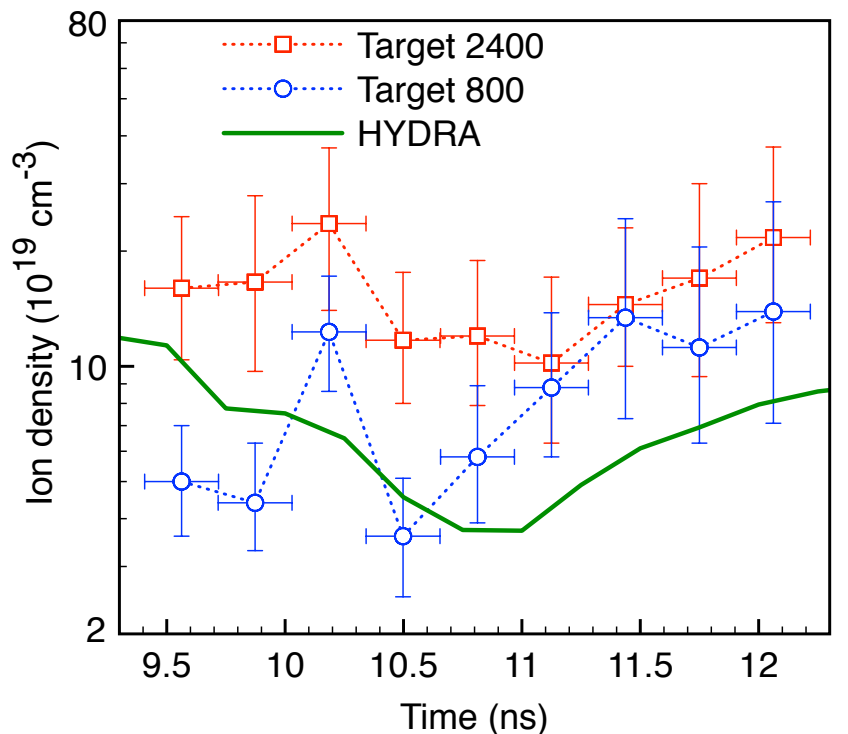

FIG. 7. Ion density of both targets obtained from their $w / y$ ratio, assuming a cylindrical shape, compared with the predictions from HYDRA. The uncertainty bars come from the width of the temperature distributions, as well as the error in the determination of the $w / y$ ratio.

the experiment. It can be seen that the hydrodynamic simulations agree by a factor of $<2$ within error bars with the experimental results for the ion density. Note how both the simulations and our results show similar trends, with the ion density increasing from $11 \mathrm{~ns}$ on, although in each case this increase starts at a slightly different time, showing some small differences between both dots.

Furthermore, after $t=11 \mathrm{~ns}$, both targets have the same density within error bars. This is particularly interesting, because it is from that moment on that the thickness of Target 800 is known whereas the results obtained for the density of Target 800 before $11 \mathrm{~ns}$ rely on the assumption that the dot is not expanding. For the times when the thicknesses of both targets are known, both of them have the same density. This is in agreement with both dots reaching equilibrium with the surrounding plasma regardless of their initial conditions, and therefore being a good diagnostic of the conditions of the plasma inside the hohlraum.

For earlier times, there is a clear difference between the ion density values obtained for both shots (up to a factor of 3). Although this might be an actual physical effect, as the dots are initially different, there is another possibility that needs consideration. During this analysis, it has been assumed that neither of the targets expanded axially during the peak power of the laser pulse, an approximation based on the XRPINH results for Target 2400, whose thickness changed only by $\pm 14 \%$ during the this time. However, it can be the case that Target 800 is expanding faster than Target 2400 during those early times and its thickness changes more. For a given temperature and density, a smaller $H / R$ quotient corresponds to a greater $w / y$ ratio in the axially emitted spectrum 26 . If the thickness of Target 800 at early times were smaller than what has been assumed, the constant-density lines in figure 6 would be shifted upwards for the timesteps before $11 \mathrm{~ns}$, in the case of Target 800, which would translate in a higher ion density for the first timesteps. In that case, an early-time dot-to-dot density comparison would reveal a reduced contrast, further suggesting a more rapid equilibration. Nevertheless, with the information available from these shots, it is difficult to confirm or rule out this possibility, and additional data of the dots' expansion during the early times of the experiment needs to be collected to check whether the dots reach equilibrium with the surrounding plasma during the early times of their expansion.

\section{A. Absolute flux}

The ion density values obtained using this new method are in reasonable agreement with the predictions from the hydrodynamic simulations. However, it is still necessary to check whether they are consistent with other experimental data. This can be done in this particular platform, as the data from the NXS are absolutely calibrated, and so the experimental spectra are measured in units of $\mathrm{J} / \mathrm{keV} / \mathrm{ns} / \mathrm{sr}$. In addition, the Cretin simulations return the spectra measured at the detector in absolute units as well and therefore we can compare the measured flux in absolute units with the results from the Cretin simulations at the measured ion densities 36 . This comparison is particularly significant because the absolut flux $F$ depends on the ion density as

$$
F \propto n_{i}^{2} V=n_{i} N
$$

and, given that in this experiments the total number of ions $N$ is fixed for each dot, the absolute flux is directly proportional to the ion density of the target.

Figure 8 shows one example of this result for each target. The spectra shown in this figure were taken at $t=11.1 \mathrm{~ns}$. The solid lines correspond to the experimental values of the spectral flux, where a $20 \%$ error bar has been included to account for the uncertainty in the absolute calibration. The shaded areas correspond to the absolute flux predicted by the simulations. The extent of this area arises from the width in the measured temperature distribution and the uncertainty in the density, as the greater the values of these parameters, the greater the flux obtained from the Cretin model. It can be seen that the results from the computational model agree with the experimental spectra well within error bars. The agreement in the absolute flux between simulations and experiment is within a factor of two for the whole duration of the peak power of the pulse for both dots.

These results show that the ion density values presented in figure 7 are consistent with the experimental 


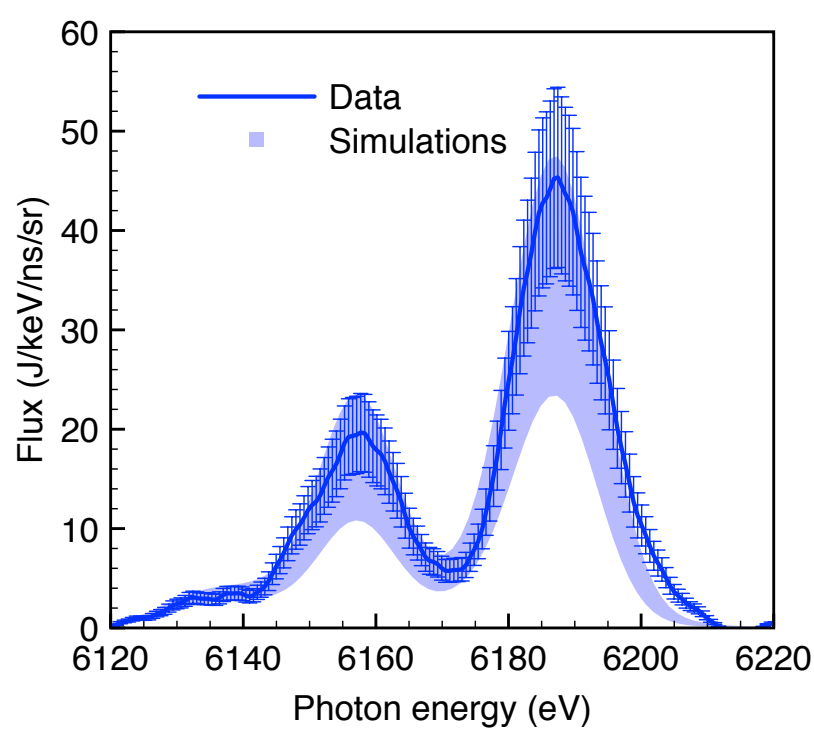

(a)

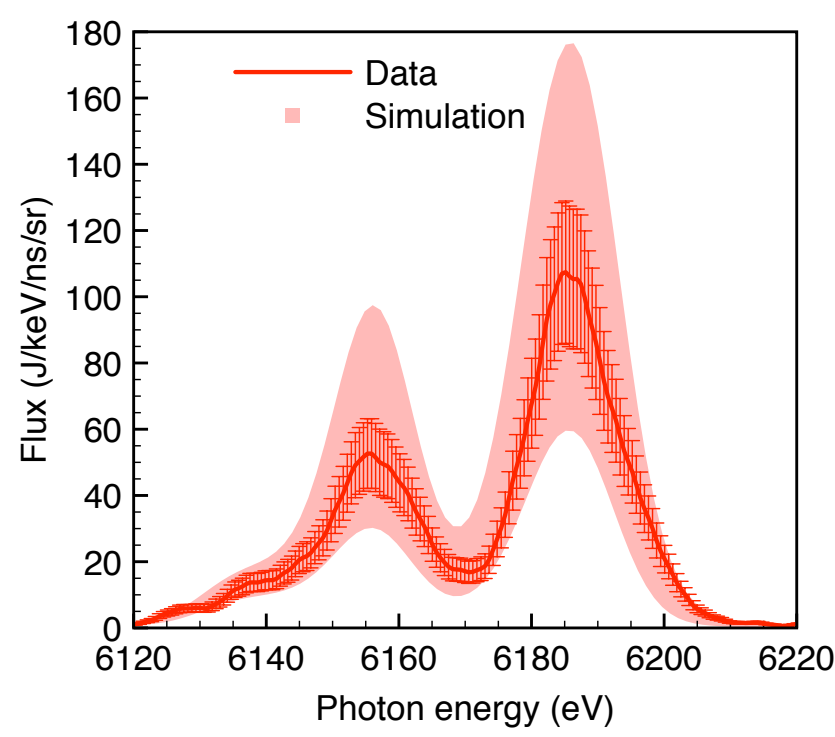

(b)

FIG. 8. Comparison between the absolute flux at $t=11.1 \mathrm{~ns}$ measured in the experiment (solid lines) and the result from the cylindrical model for the obtained temperatures and densities (shaded regions) for Target 800 (8a) and Target 2400 (8b). A $20 \%$ error bar has been included in the experimental data to account for the uncertainty in the NXS calibration. The width of the shaded regions shows the range of values of the absolute flux predicted by the simulations within the uncertainty in the temperature and density measurements.

values of the absolute flux, demonstrating that the geometric modification of the $w / y$ line ratio in cylindrical plasmas can be used as a new spectroscopic diagnostic of the ion density. The fact that the experimental values and those from a 'perfect cylinder' assumption agree, indicates that the error introduced by this assumption is not significant.

\section{B. Results for spherical model}

So far, it has been shown how the temperature and $w / y$ ratios extracted from the experimental data can be coupled with the cylindrical model that was benchmarked at the OMEGA experiments to obtain a measurement of the ion density of the plasma. However, it is still unclear how important it is to treat the plasma as a cylinder, and not as an isotropic system. In Pérez-Callejo et al. ${ }^{26}$ it was shown how for the experimental conditions achieved at OMEGA, the $w / y$ ratios for the axial view of a cylinder were similar to those obtained for a sphere. For this reason, one might ask what the inferred density would be if a sphere were assumed instead of a cylinder in this case.

To answer this question, the same procedure was followed using a spherical model instead. In this case, for each value of $n_{i}$ in the grid, the radius of the sphere was calculated as

$$
R\left(n_{i}\right)=\left(\frac{3 N}{4 \pi n_{i}}\right)^{1 / 3}
$$

to force conservation of particles. Note that in this case, this approximation is not consistent with the experimental measurements, as the measured thickness of the targets is not taken into account. By calculating what the $w / y$ ratio would be for a spherical plasma (instead of a cylinder, as in the process described above) at a given temperature and density, a different $w / y$ map, equivalent to that in figure 6 can be constructed for each target. By interpolating the experimental values again, analogous values of the ion density are inferred. Figure 9 shows the results for the ion density obtained for both targets when assuming a spherical plasma. The results from a cylindrical model and the hydrodynamic predictions are also shown for comparison and the scale of the y-axis has been increased to show all the data. Although for Target 2400 the spherical predictions are in agreement with the results from the cylindrical model (within error bars), in the case of Target 800, there is a difference of one order of magnitude between the results for both models.

In the previous section we showed that the cylindrical simulations return values of the ion densities that lead to predictions of the spectral flux that compare favorably with the measurements. Given that the flux is directly proportional to the ion density, the values of the spectral flux of Target 800 obtained with a spherical model are approximately one order of magnitude lower than those obtained with the cylindrical model, which is not consistent with the experimental data. Although depending on the initial thickness of the dot, the ion density results obtained from both models might agree, as it is the case 


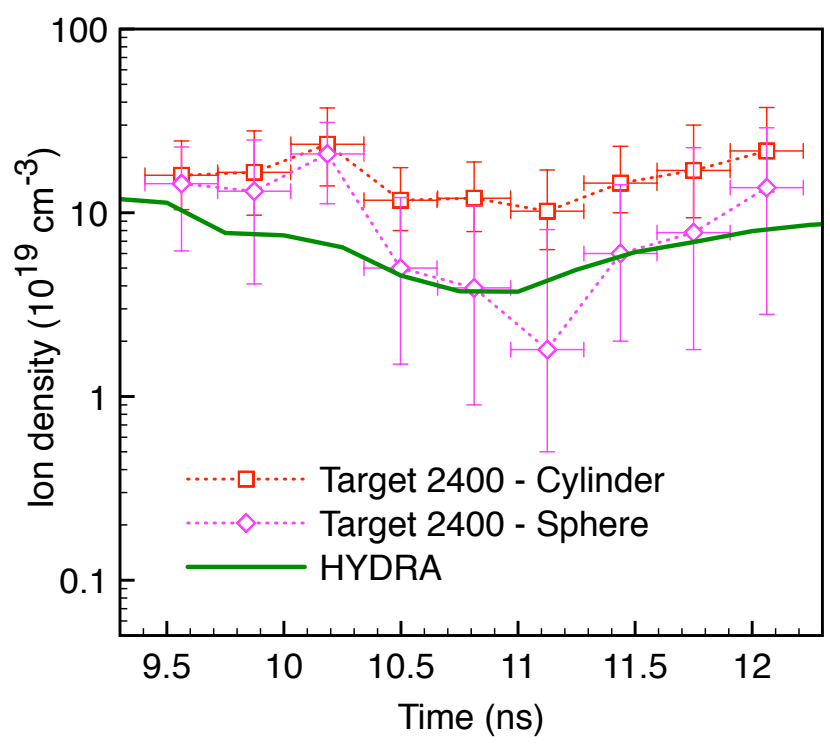

(a)

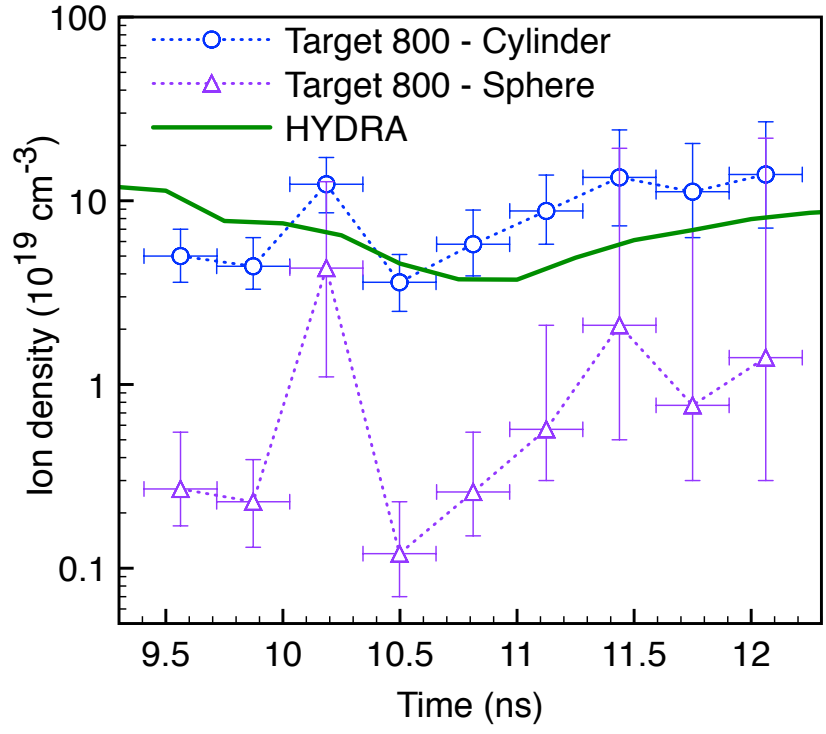

(b)

FIG. 9. Results for the experimentally inferred ion density using the spherical model for Target 2400 9a and Target 800 (9b), compared with the corresponding results from the cylindrical model and the HYDRA simulations.

for Target 2400, the disagreement for Target 800 shows that the spherical model is not reliable in a general case.

\section{CONCLUSIONS AND SUGGESTIONS FOR FUTURE WORK}

The work presented here shows how the effects of the plasma geometry on the spectra can be used as an additional spectroscopic diagnostic, providing useful information about the optical depth and hence the density of the plasma. By combining the predictions for the $w / y$ ratio from experimentally-benchmarked multidimensional radiative transfer models, with X-ray imaging and spectroscopic data from cylindrical tracers in ICF experiments we have been able to obtain time-resolved values of the ion density inside the hohlraum during an implosion. These are the first results of time-resolved coupled temperature and density measurements inside a hohlraum during an ICF experiment, which shows the importance and usefulness of this method. However, more work should be done to ensure a full characterization of the platform and study the surrogacy of the dot tracers, which will lead to more meaningful measurements of the ion density inside the hohlraum.

It was also shown that if the same process is followed using a spherical model and not taking into account the fact that the tracers are cylindrical, the predicted line ratios result in the wrong density being inferred, and the results depend on the initial thickness of the dot. Differences of up to an order of magnitude in the ion density are obtained, yielding a disagreement between the experimental and simulated spectral fluxes. This implies that a spherical model is not reliable in a general case, as it overlooks the spectral changes due to the plasma geometry.

We also showed how for the two cases of study the calculated ion densites agree after $t=11 \mathrm{~ns}$. This could indicate that some sort of equilibrium is reached with the surrounding plasma, thus meaning that the tracer dots can be used not only to measure the electron temperature inside the hohlraum, but also as an indicator of the particle density. Future work should include acquiring more data for different thicknesses for the whole duration of the expansion, necessary to determine whether equilibrium is being reached or not. Additionally, the behavior of the magnetic fields in the hohlraum in the presence of the dot should be experimentally studied to compare with the results from radiative-hydrodynamic simulations 37 .

\section{ACKNOWLEDGEMENTS}

G.P.-C., S. J. R. and J. S. W. gratefully acknowledge support from LLNL under grant number B617350.

This work was performed under the auspices of the U.S. Department of Energy by Lawrence Livermore National Laboratory under Contract DE-AC52-07NA27344. This document was prepared as an account of work sponsored by an agency of the United States government. Neither the United States government nor Lawrence Livermore National Security, LLC, nor any of their employees makes any warranty, expressed or implied, or assumes any legal liability or responsibility for the accuracy, completeness, or usefulness of any information, apparatus, 
product, or process disclosed, or represents that its use would not infringe privately owned rights. Reference herein to any specific commercial product, process, or service by trade name, trademark, manufacturer, or otherwise does not necessarily constitute or imply its endorsement, recommendation, or favoring by the United States government or Lawrence Livermore National Security, LLC. The views and opinions of authors expressed herein do not necessarily state or reflect those of the United States government or Lawrence Livermore National Security, LLC, and shall not be used for advertising or product endorsement purposes.

\section{DATA AVAILABILITY STATEMENT}

The data that support the findings of this study are available from Lawrence Livermore National Laboratory. Restrictions apply to the availability of these data, which were used under license for this study. Data are available from the authors upon reasonable request and with the permission of Lawrence Livermore National Laboratory.

\section{REFERENCES}

${ }^{1}$ E. Storm, "Approach to high compression in inertial fusion," $J$. Fusion Energy, vol. 7, no. 2, pp. 131-137, 1988.

${ }^{2}$ J. Lindl, "Development of the indirect-drive approach to inertial confinement fusion and the target physics basis for ignition and gain," Phys. Plasmas, vol. 2, no. 11, pp. 3933-4024, 1995.

${ }^{3}$ R. E. Olson, R. J. Leeper, A. Nobile, J. A. Oertel, G. A. Chandler, K. Cochrane, S. C. Dropinski, S. Evans, S. W. Haan, J. L. Kaae, J. P. Knauer, K. Lash, L. P. Mix, A. Nikroo, G. A. Rochau, G. Rivera, C. Russell, D. Schroen, R. J. Sebring, D. L. Tanner, R. E. Turner, and R. J. Wallace, "Shock propagation, preheat, and x-ray burnthrough in indirect-drive inertial confinement fusion ablator materials," Phys. Plasmas, vol. 11, no. 5, pp. 27782789, 2004.

${ }^{4}$ O. S. Jones, L. J. Suter, H. A. Scott, M. A. Barrios, W. A. Farmer, S. B. Hansen, D. A. Liedahl, C. W. Mauche, A. S. Moore, M. D. Rosen, J. D. Salmonson, D. J. Strozzi, C. A. Thomas, and D. P. Turnbull, "Progress towards a more predictive model for hohlraum radiation drive and symmetry," Phys. Plasmas, vol. 24, no. 5, p. $056312,2017$.

${ }^{5}$ L. Heroux, "A spectroscopic determination of electron temperature in a high temperature plasma," Proceedings of the Physical Society, vol. 83, no. 1, p. 121, 1964.

${ }^{6}$ J. D. Kilkenny, R. W. Lee, M. H. Key, and J. G. Lunney, "X-ray spectroscopic diagnosis of laser-produced plasmas, with emphasis on line broadening," Phys. Rev. A ., vol. 22, pp. 2746-2760, Dec. 1980.

${ }^{7}$ J. P. Apruzese, K. G. Whitney, J. Davis, and P. C. Kepple, "Kshell line ratios and powers for diagnosing cylindrical plasmas of neon, aluminum, argon, and titanium," J. Quant. Spectrosc. Radiat. Transfer, vol. 57, no. 1, pp. 41 - 61, 1997.

${ }^{8}$ J. P. Apruzese, D. Duston, and J. Davis, "K-shell aluminum resonance line ratios for plasma diagnosis using spot spectroscopy," J. Quant. Spectrosc. Radiat. Transfer, vol. 36, no. 4, pp. $339-$ 344, 1986.

${ }^{9}$ R. S. Marjoribanks, M. C. Richardson, P. A. Jaanimagi, and R. Epstein, "Electron-temperature measurement in laserproduced plasmas by the ratio of isoelectronic line intensities," Phys. Rev., vol. 46, Aug. 1992.
${ }^{10}$ C. J. Keane, B. A. Hammel, D. R. Kania, J. D. Kilkenny, R. W. Lee, A. L. Osterheld, L. J. Suter, R. C. Mancini, C. F. Hooper, and N. D. Delamater, "X-ray spectroscopy of high-energy density inertial confinement fusion plasmas," Phys. Fluids B: Plasma Phys., vol. 5, no. 9, pp. 3328-3336, 1993.

${ }^{11}$ C. A. Back, D. H. Kalantar, R. L. Kauffman, R. W. Lee, B. K. MacGowan, D. S. Montgomery, L. V. Powers, T. D. Shepard, G. F. Stone, and L. J. Suter, "Measurements of electron temperature by spectroscopy in hohlraum targets," Phys. Rev. Lett., vol. 77 , no. 21,1996 .

${ }^{12}$ T. D. Shepard, C. A. Back, D. H. Kalantar, R. L. Kauffman, C. J. Keane, D. E. Klem, B. F. Lasinski, B. J. MacGowan, L. V. Powers, L. J. Suter, R. E. Turner, B. H. Failor, and W. W. Hsing, "Isoelectronic x-ray spectroscopy to determine electron temperatures in lon-scale-length inertial-confinement-fusion plasmas," Phys. Rev. E, vol. 53, no. 5, 1996.

${ }^{13}$ M. A. Barrios, D. A. Liedahl, M. B. Schneider, O. Jones, G. V. Brown, S. P. Regan, K. B. Fournier, A. S. Moore, J. S. Ross, O. Landen, R. L. Kauffman, A. Nikroo, J. Kroll, J. Jaquez, H. Huang, S. B. Hansen, D. A. Callahan, D. E. Hinkel, D. Bradley, and J. D. Moody, "Electron temperature measurements inside the ablating plasma of gas-filled hohlraums at the National Ignition Facility," Phys. Plasmas, vol. 23, no. 5, p. 056307, 2016.

${ }^{14}$ M. A. Barrios, J. D. Moody, L. J. Suter, M. Sherlock, H. Chen, W. Farmer, J. Jaquez, O. Jones, R. L. Kauffman, J. D. Kilkenny, J. Kroll, O. L. Landen, D. A. Liedahl, S. A. Maclaren, N. B. Meezan, A. Nikroo, M. B. Schneider, D. B. Thron, K. Widmann, and G. Pérez-Callejo, "Developing an experimental basis for understanding transport in NIF hohlraum plasmas," Phys. Rev. Lett., 2018.

${ }^{15}$ G. H. Miller, E. I. Moses, and C. R. Wuest, "The National Ignition Facility," Opt. Eng., vol. 43, pp. 43 - 43 - 13, 2004.

${ }^{16}$ M. M. Marinak, R. E. Tipton, O. L. Landen, T. J. Murphy, P. Amendt, S. W. Haan, S. P. Hatchett, C. J. Keane, R. McEachern, and R. Wallace, "Three-dimensional simulations of nova high growth factor capsule implosion experiments," Physics of Plasmas, vol. 3, no. 5, pp. 2070-2076, 1996.

${ }^{17}$ F. M. Kerr, S. J. Rose, J. S. Wark, and F. P. Keenan, "Enhancement of optically thick to thin line intensities in solar and stellar coronal plasmas through radiative transfer effects: an angularly resolved study," Astrophys. J., vol. 613, pp. L181-L184, Oct. 2004.

${ }^{18}$ F. M. Kerr, S. J. Rose, and J. S. Wark, "An analytic geometryvariant approach to line ratio enhancement above the optically thin limit," Astrophys. J., vol. 629, p. 1091, Jan. 2005.

${ }^{19}$ F. M. Kerr, A. Gouveia, O. Renner, S. J. Rose, H. A. Scott, and J. S. Wark, "Line radiation effects in laboratory and astrophysical plasmas," J. Quant. Spectrosc. Radiat. Transfer, vol. 99, no. 1, pp. 363-369, 2006.

${ }^{20}$ P. A. M. Dirac, The Collected Works of P. A. M. Dirac 19241948, ch. Approximate rate of neutron multiplication for a solid of arbitrary shape and uniform density, I: General theory, pp. 1115-1128. Oxford University Press, 1995.

${ }^{21}$ P. A. M. Dirac, K. Fuchs, R. Peierls, , and P. Preston, The Collected Works of P. A. M. Dirac 1924-1948, ch. Approximate rate of neutron multiplication for a solid of arbitrary shape and uniform density, II: Application to the oblate spheroid, hemisphere and oblate hemispheroid, pp. 1129-1145. Oxford University Press, 1995.

${ }^{22}$ D. M. Chambers, P. A. Pinto, J. Hawreliak, I. R. Al'Miev, A. Gouveia, P. Sondhauss, E. Wolfrum, J. S. Wark, S. H. Glenzer, R. W. Lee, P. E. Young, O. Renner, R. S. Marjoribanks, and S. Topping, "K-shell spectroscopy of an independently diagnosed uniaxially expanding laser-produced aluminum plasma," Phys. Rev. E, vol. 66, p. 026410, Aug 2002.

${ }^{23}$ F. P. Keenan, J. G. Doyle, M. S. Madjarska, S. J. Rose, L. A. Bowler, J. Britton, L. McCrink, and M. Mathioudakis, "Intensity enhancement of o vi ultraviolet emission lines in solar spectra due to opacity," Astrophys. J., vol. 784, 2014. 
${ }^{24} \mathrm{P}$. Hatfield, "Using line intensity ratios to determine the geometry of plasma in stars via their apparent areas," High Energy Density Phys., vol. 6, no. 3, pp. $301-304,2010$.

${ }^{25}$ T. R. Boehly, D. L. Brown, R. S. Craxton, R. L. Keck, J. P. Knauer, J. H. Kelly, T. J. Kessler, S. A. Kumpan, S. J. Loucks, S. A. Letzring, F. J. Marshall, R. L. McCrory, S. F. B. Morse, W. Seka, J. M. Soures, and C. P. Verdon, "Initial performance results of the OMEGA laser system," Opt. Commun., vol. 133, no. 1 , pp. $495-506,1997$.

${ }^{26}$ G. Pérez-Callejo, L. C. Jarrott, D. A. Liedahl, E. V. Marley, G. E. Kemp, R. F. Heeter, J. A. Emig, M. E. Foord, K. Widmann, J. Jaquez, H. Huang, S. J. Rose, J. S. Wark, and M. B. Schneider, "Laboratory measurements of geometrical effects in the x-ray emission of optically thick lines for icf diagnostics," Physics of Plasmas, vol. 26, no. 6, p. 063302, 2019.

${ }^{27}$ A. H. Gabriel, "Dielectronic satellite spectra for highly-charged helium-like ion lines," Mon. Not. R. Astron. Soc., vol. 160, pp. 99-119, 1972.

${ }^{28}$ H. A. Scott, "CRETIN - a radiative transfer capability for laboratory plasmas," J. Quant. Spectrosc. Radiat. Transfer, vol. 71, no. 2, pp. 689 - 701, 2001. Radiative Properties of Hot Dense Matter.

${ }^{29}$ G. Pérez-Callejo, D. A. Liedahl, M. B. Schneider, S. J. Rose, and J. S. Wark, "The use of geometric effects in diagnosing ion density in ICF-related dot spectroscopy experiments," High Energy Density Physics, vol. 30, pp. 45 - 51, 2019.

${ }^{30}$ G. Pérez-Callejo, J. Wark, and S. Rose, "Radiation transfer in cylindrical, toroidal and hemi-ellipsoidal plasmas," Journal of Quantitative Spectroscopy and Radiative Transfer, vol. 235C, pp. 24-30, 2019.
${ }^{31}$ S. MacLaren, M. Schneider, K. Widmann, J. Hammer, B. Yoxall, J. Moody, P. Bell, L. Benedetti, D. Bradley, M. Edwards, et al., "Novel characterization of capsule x-ray drive at the national ignition facility," Physical review letters, vol. 112, no. 10, p. 105003, 2014.

${ }^{32}$ F. Pérez, G. E. Kemp, S. P. Regan, M. A. Barrios, J. Pino, H. Scott, S. Ayers, H. Chen, J. Emig, J. D. Colvin, M. Bedzyk, M. J. Shoup, A. Agliata, B. Yaakobi, F. J. Marshall, R. A. Hamilton, J. Jaquez, M. Farrell, A. Nikroo, and K. B. Fournier, "The NIF x-ray spectrometer calibration campaign at Omega," Review of Scientific Instruments, vol. 85, no. 11, p. 11D613, 2014.

${ }^{33}$ E. V. Marley, D. A. Liedahl, M. B. Schneider, R. F. Heeter, L. C. Jarrott, C. W. Mauche, G. E. Kemp, M. E. Foord, Y. Frank, K. Widmann, and J. Emig, "Using l-shell x-ray spectra to determine conditions of non-local thermal dynamic equilibrium plasmas," Review of Scientific Instruments, vol. 89, no. 10, p. 10F106, 2018.

${ }^{34}$ J. H. Holland, "Adaptation in natural and artificial systems," Ann Arbor: University of Michigan Press, 1975.

${ }^{35}$ S. B. Hansen, J. Bauche, C. Bauche-Arnoult, and M. F. Gu, "Hybrid atomic models for spectroscopic plasma diagnostics," High Energy Density Phys., vol. 3, no. 1, pp. 109 - 114, 2007. Radiative Properties of Hot Dense Matter.

${ }^{36}$ The authors benchmarked the absolute units for the flux from the Cretin model against the spectra from aluminium microdots presented in Chambers et al ${ }^{22}$.

${ }^{37}$ W. Farmer, O. Jones, M. Barrios, D. Strozzi, J. Koning, G. Kerbel, D. Hinkel, J. Moody, L. Suter, D. Liedahl, et al., "Heat transport modeling of the dot spectroscopy platform on nif," Plasma Physics and Controlled Fusion, vol. 60, no. 4, p. 044009, 2018. 